\title{
New Insight into the Toughening Mechanisms of Seashell: From Arch Shape to Multilayer Structure
}

\author{
Quan Yuan, ${ }^{1}$ Botao Chen, ${ }^{1}$ Bin Chen, ${ }^{2}$ and Zeyun Wang ${ }^{1}$ \\ ${ }^{1}$ School of Architecture and Civil Engineering, Xihua University, Hongguang Town, Chengdu 610039, China \\ ${ }^{2}$ College of Aerospace Engineering, Chongqing University, Shapingba District, Chongqing 400044, China \\ Correspondence should be addressed to Quan Yuan; yuanqxh@163.com
}

Received 25 April 2016; Accepted 2 August 2016

Academic Editor: Christian Kübel

Copyright (c) 2016 Quan Yuan et al. This is an open access article distributed under the Creative Commons Attribution License, which permits unrestricted use, distribution, and reproduction in any medium, provided the original work is properly cited.

\begin{abstract}
A seashell is a closed three-dimensional curved surface formed by two symmetrical open shells. Three-point bending is performed on a pure aragonite straight beam (PASB) model and a multilayer structure curved beam (MSCB) model to elucidate the structureproperty relationships of seashells. The integrity of the PASB is broken because of the introduction of a soft layer, but this drawback is compensated by the peculiar arch shape and the internal multilayer structure. The effective modulus, stiffness, and fracture energy of MSCB increase with an increase in volume fraction, aspect ratio of aragonite platelet, overlap ratio of hard layers, and ratio of the elastic modulus of the hard layer to the shear modulus of the soft layer. New design disciplines drawn from the MSCB model are peculiar arch shape, internal multilayer structure of larger volume fraction, and aspect ratio of hard layers and nanoscaled soft layers.
\end{abstract}

\section{Introduction}

Nacre, which is the inner layer of seashells, is a lamellar bioceramic material composed of brittle aragonite platelets (hard phase) closely staggered and bonded together by less tough proteins (soft phase) [1]. Seashells exhibit 2-3 levels of structural hierarchy and 2-3 orders of toughness amplification [2]. More than 10 strengthening and toughening mechanisms have been identified to contribute to the energy dissipation in nacre; these mechanisms include crack deflection, pull-out of hard phase, mineral bridge, and multicrack propagation [3]. The strengthening and toughening design disciplines for structural ceramics from seashell reveal that fracture energy and flaw tolerance can be increased by maintaining high strength and stiffness as well as by overcoming the intrinsic fault of the brittle fracture [4]. Nacreous bioceramics fabricated by mimicking the lamellar structure are stronger and tougher than their structural components $[5,6]$; however, no material has matched the strength and toughness amplification of nacre. One reason is that the ideal combination of stiffness, strength, and toughness is difficult to achieve by mimicking the structure at one level only. Therefore, a multiscale micromechanical model is needed to elucidate the structure-property relationships of seashells.
Research posits that a high mechanical performance is associated with the microscopic brick-mortar microstructure, mineral bridge, and nanoasperities of nacre [7-9]. Macroscopic nacre has a three-dimensional curved surface, and the cross section of a shell has an arch shape. Shells are typically subjected to loads perpendicular to the surface. Compressive resistance plays an important role in force transfer. In this study, a simple three-point bending multilayer curved beam is proposed to investigate the strengthening and toughening mechanisms of shells. The effects of arch shape and multilayer structure on stiffness, strength, and toughness are studied. New design disciplines drawn from the model are useful for enhancing the fabrication of bioceramics.

\section{Bending Stiffness and Strength of Arch Nacre}

A seashell is a closed three-dimensional curved surface formed by two symmetrical open shells. Except for the reinforcement of the ribs on the surface, the arch shape meets the strength requirements of shells. The fracture section of nacre is shown in Figure 1. The aragonite platelets are stacked in the thickness direction, forming a peculiar 


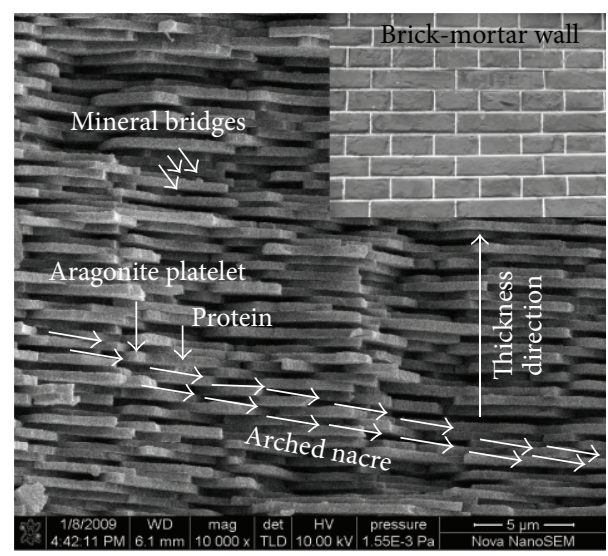

(a) Brick-mortar structure of nacre

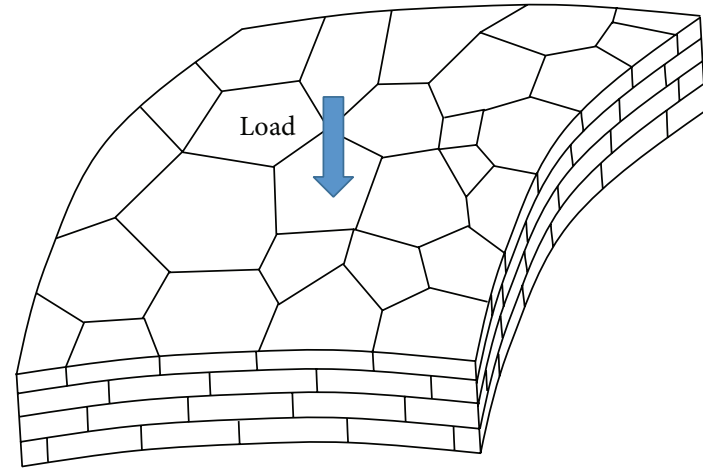

(b) Schematic of the curved surface of nacre

FIGURE 1: Fracture section of a seashell and arch-shaped nacre subjected to an external load in the thickness direction.

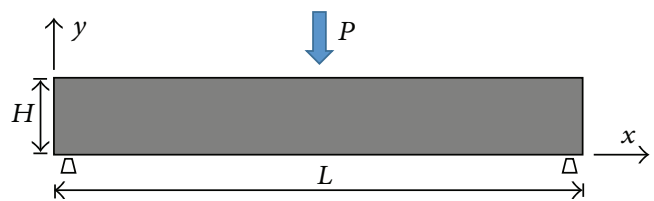

(a) PASB model

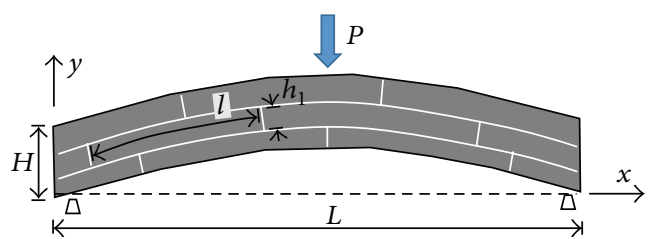

(b) MSCB model

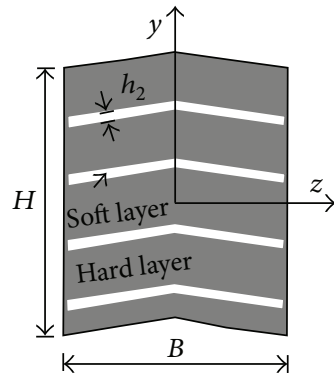

(c) Cross section of MSCB

FIgURE 2: PASB and MSCB.

"brick-mortar" microstructure (see the arrangements in the inset in Figure 1(a)). Mineral bridges and nanoasperities can be observed on the surface of aragonite platelets. As shown, the fracture is rough and uneven, and the aragonite platelet arranging trend (white arrows) tends toward the thickness direction. The inner layer of nacre is as curved as the surface shape of the seashell (Figure 1(b)). When an external load is transferred to the arch nacre, the neighboring aragonite platelets are compressed. An arch-shaped multilayer beam model is developed to estimate the compressed stiffness and bending strength of nacre.

Mineral bridges endow arch nacre with inherent mechanical robustness. The "brick-mortar" microstructure can be modeled as a multilayer structure because of the large aspect ratio of aragonite platelets. The three-point bending of the pure aragonite straight beam (PASB) and multilayer structure curved beam (MSCB) models is shown in Figure 2. PASB and MSCB represent the brittle material to be strengthened and the strengthened material, respectively. The PASB and the MSCB have a height of $H$, a length of $H$, and a width of $B$. The thicknesses of the hard and soft layers in the MSCB are $h_{1}$ and $h_{2}$, respectively. For the PASB, the elastic modulus and Poisson ratio of the hard layer are $E_{1}$ and $v_{1}$, and the shear modulus and Poisson ratio of the soft layer are $G$ and $v_{2}$. By adopting the coordinate system shown in Figure 2(a), the bending moment, shear force, and axial force acting on a cross section of the PASB are as follows:

$$
\begin{aligned}
& M_{0}=M_{0}(x)=\frac{P}{2} x \quad\left(0 \leq x \leq \frac{L}{2}\right), \\
& Q_{0}=\frac{P}{2}, \\
& N_{0}=0,
\end{aligned}
$$

where $x$ and $P$ denote the position coordinate of cross section and the external force. Under the assumption that the axial equation of the prismatic curved beam (Figure 2(b)) is $y=$ $f(x)$, the internal forces on the cross section of MSCB are as follows [10]:

$$
\begin{aligned}
& M=M(x)=M_{0}, \\
& Q=Q(x)=Q_{0} \cos \varphi, \\
& N=-Q_{0} \sin \varphi,
\end{aligned}
$$

where $\varphi$ is the angle between the axis of MSCB and the $x$ axis and $\tan \varphi=f^{\prime}(x)$. Equations (1c) and (2c) show that pressures emerge on the cross section of MSCB.

Soft protein is assumed to carry only shear stresses because of its lower bending stiffness and strength compared 
with that of hard aragonite platelets. The effective modulus of MSCB can be obtained from the tension-shear chain model [11]:

$$
\frac{E}{E_{1}}=\frac{(1-\Phi) \Phi \rho^{2} V_{f}^{2}}{\left(1-V_{f}\right) \lambda+(1-\Phi) \Phi \rho^{2} V_{f}}
$$

where $\lambda=E_{1} / G_{2}, V_{f}=h_{1} /\left(h_{1}+h_{2}\right)$, and $\rho=l / h_{1} ; V_{f}, \rho$, and $\Phi$ denote the volume fraction, the aspect ratio, and the overlap ratio of the hard layers. As shown in (3), $E / E_{1}=1$ if $V_{f}=1$ and MSCB degenerates into PASB. The bending stiffness is dependent on the geometry of the cross section. For the cross section of MSCB shown in Figure 2(c), the moment of inertia and the maximum static moment are as follows $[12,13]$ :

$$
\begin{aligned}
I_{z} & =2 \int_{\Omega} y^{2} d A, \\
S_{z \max } & =\int_{\Omega} y d A,
\end{aligned}
$$

where $\Omega$ is the area of the hard layers (gray area in Figure 2(c)). PASB becomes lamellated after the soft layers are introduced. The number of hard layers under the invariance of $H, h_{1}$, and $V_{f}$ can be obtained as follows:

$$
n=\left(\frac{H}{h_{2}}-1\right) V_{f}+1 \text {. }
$$

In consideration of the integration in every hard layer, (4a) and (4b) can be expressed as follows:

$$
\begin{aligned}
I_{z} & =\frac{B H}{12}\left[\left(H-h_{2}\right)\left(H-2 h_{2}\right) V_{f}+\left(3 H-2 h_{2}\right) h_{2}\right] \\
S_{z \max } & =\frac{B}{8}\left[4 H h_{2}-4 h_{2}^{2}+\left(H-h_{2}\right)^{2} V_{f}\right. \\
& \left.-\frac{2}{V_{f}}\left(H-h_{2}\right) h_{2}\right] .
\end{aligned}
$$

Similarly, $I_{z}=B H^{3} / 12=I_{z 0}, S_{z \max }=B H^{2} / 8=S_{z 0}$, and MSCB degenerate into PASB if $V_{f}=1$. The bending stiffness ratio of MSCB to PASB is as follows:

$$
\kappa=\frac{E I_{z}}{E_{1} I_{z 0}} .
$$

The rupture model of the PASB is a brittle bending fracture, and interfacial failure is not involved in MSCB. By applying Euler-Bernoulli beam theory, the normal stress and the shear stress on the cross section of MSCB can be obtained as follows:

$$
\begin{aligned}
\sigma_{\max } & =\frac{M H}{2 I_{z}}-\frac{N}{A V_{f}}, \\
\tau_{\max } & =\frac{S_{z \max } Q}{I_{z} B} .
\end{aligned}
$$

Substituting (2a)-(2c) into the above equation yields the following:

$$
\begin{aligned}
\sigma_{\text {max }} & =\frac{P H x}{4 I_{z}}-\frac{P \sin \varphi}{2 A V_{f}}, \\
\tau_{\max } & =\frac{P S_{z \max } \cos \varphi}{2 I_{z} B} .
\end{aligned}
$$

Equations (9a) and (9b) show that the maximum normal stress of the hard layer and the maximum shear stress of the soft layer are both achieved at the center of the beam $(x=L / 2)$. The critical load carried by MSCB depends on which layer first achieves the ultimate strength; thus, we have the following equation:

$$
\frac{\sigma_{\max }}{\sigma_{b}} \geq \frac{\tau_{\max }}{\tau_{s}},
$$

where $\sigma_{b}$ and $\tau_{s}$ are the tension strength of the hard layer and the shear strength of the soft layer, respectively. The invariance of $\sigma_{b}$ may hold and $\tau_{s}$ is devisable in general materials. For the strongly bonded interface, MSCB produces brittle fracture if the maximum normal stress achieves the tension strength of the hard layer:

$$
\sigma_{\max }=\frac{P H L}{8 I_{z}}=\sigma_{b} .
$$

In this case, the external load is

$$
P_{1}=\frac{8 I_{z} \sigma_{b}}{H L} .
$$

Using a similar derivation, the critical load carried by PASB is $P_{0}=8 I_{z 0} \sigma_{b} / H L$. For the weakly bonded interface, MSCB produces interfacial failure if the maximum shear stress achieves the interfacial strength of the soft layer:

$$
\tau_{\max }=\frac{P S_{z \max }}{2 I_{z} B}=\tau_{s} .
$$

In this case, the external load is

$$
P_{2}=\frac{2 I_{z} \tau_{s} B}{S_{z \max }}
$$

The critical load is $[P]=\min \left(P_{1}, P_{2}\right)$. The bending strength ratio of MSCB to PASB can be defined as follows:

$$
\chi=\frac{[P]}{P_{0}} \text {. }
$$

The soft layer is assumed to exhibit an elastic-ideal plastic behavior. When soft protein begins to yield, plastic deformations proceed along the layers. With an increase in the external load, the normal stress can achieve the compressive strength (also denoted by $\sigma_{b}$ ) at the center of MSCB. By combining and solving (8b) to (10), the position of the plastic section can be expressed as follows:

$$
\cos \varphi_{0}=\frac{H L B \tau_{s}}{4 S_{z \max } \sigma_{b}} .
$$




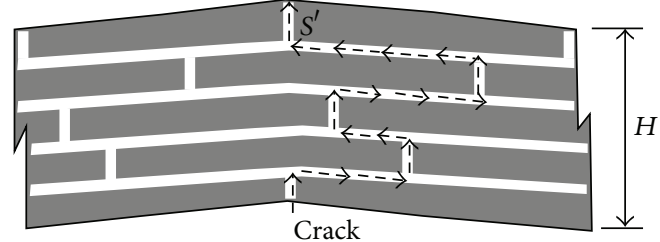

Figure 3: Crack deflection and tortuous fracture path.

If the axis of MSCB is assumed to be parabolic, that is,

$$
f(x)=\frac{4 \delta}{L^{2}}\left(x L-x^{2}\right),
$$

where $\delta$ is the arch height of MSCB, then the cosine value at the axis is

$$
\cos \varphi=\left(1+f^{\prime 2}(x)\right)^{-1 / 2} .
$$

By substituting (16) into the above equation, the length of the plastic region in MSCB can be expressed as follows:

$$
L_{c}=L-\frac{L^{2}}{4 \delta} \sqrt{\frac{16 S_{z \max }^{2} \sigma_{b}^{2}}{H^{2} L^{2} B^{2} \tau_{s}^{2}}-1 .}
$$

\section{Work of Fracture of Arch Nacre}

The integrity of the PASB is broken because of the introduction of a soft layer. If the fraction of the hard layer is sufficiently large, the bending stiffness and bending strength of MSCB become slightly influenced by the weak interface. Assume that PASB and MSCB have a single crack at the lower edge. The crack advances in a zigzag manner through the weak interface of MSCB. The path is a polyline because of the crack deflection and crack branching at the vertical interface (Figure 3). For simplicity, the work of fracture of the PASB and MSCB is calculated from the length of crack path.

The crack advances vertically throughout the cross section, causing PASB to produce a brittle fracture. The length of the crack path is equal to the height of the PASB. The fracture energy is

$$
W_{\text {PASB }}=\gamma H B \text {, }
$$

where $\gamma$ denotes the surface energy of the hard phase. Several zigzag cracks are present in the plastic region with a length of $L_{c}$. The length of a zigzag crack can be expressed as follows:

$$
S=\left(1+\frac{1}{2} \Phi \rho V_{f}\right) H
$$

By ignoring the dissipated energy of the vertical interface, the effective length of crack can be expressed as follows:

$$
S^{\prime}=S-H=\frac{1}{2} \Phi \rho V_{f} H .
$$

The number of zigzag cracks is approximate to the ratio of $L_{c}$ to $l, m=L_{c} / l$. The distribution of mineral bridges is not uniform. The fraction is approximate to $V_{b}=1 / 3$ [14]. The fracture energy of MSCB is

$$
W_{\mathrm{MSCB}}=m S^{\prime} V_{b} B \gamma=\frac{m}{6} \Phi \rho V_{f} \gamma H B .
$$

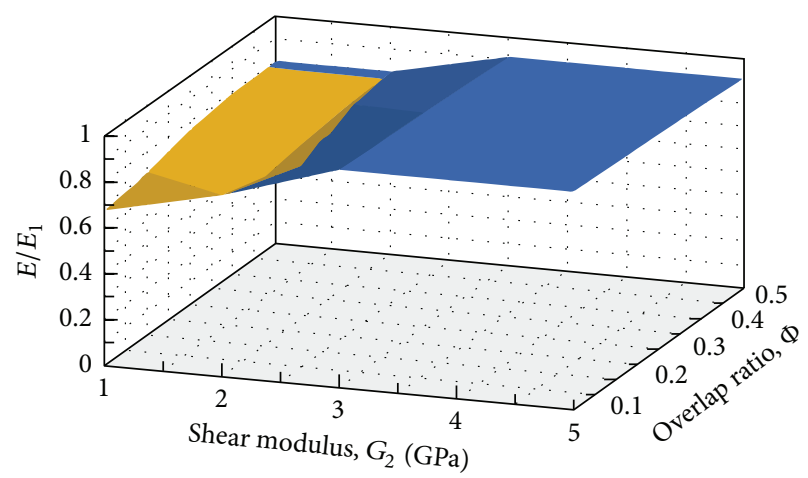

Figure 4: $E / E_{1}$ versus $G_{2}$ and $\Phi$.

\section{Results and Discussion}

The inherent mechanical robustness of the PASB is reduced because of the introduction of a soft layer; however, this drawback is compensated by the arch shape and the multilayer structure. The discrete hard layers are bounded together but are separated from one another by the soft layers (acting as adhesive layers). The geometry and property of the component phases strongly influence the bending stiffness, strength, and toughness of the MSCB. The stiffness is dependent on the effective modulus and effective moment of inertia. The effective modulus slightly decreases with the increase in the elastic modulus of the hard layer and in the shear modulus of the soft layer (3). For the MSCB, the compressive resistance of the hard phases is exploited by the arch shape. Furthermore, the multilayer structure with thin soft layers provides the MSCB with a high effective modulus. The effect of the shear modulus of the soft layers on the effective modulus is indistinguishable if the MSCB has a high hard phase content and a medium overlap ratio (Figure 4 ). The effective modulus of MSCB is mainly determined by the elastic modulus and the number of hard layers. The MSCB structure can be stiffened by the high content and aspect ratio of hard layer despite the introduction of a soft layer which is why the stiffness of MSCB can match the one of the PASB. The effective moment of inertia, which is the other factor that influences the bending stiffness, is determined by the distribution of the hard phases at the cross section of the MSCB. The bending stiffness of the MSCB decreases with the decrease in the volume fraction and the thickness of soft layers, as shown in Figure 5. If the hard phase content is adequate and constant, the influence of the thickness of the soft layers on the bending stiffness gradually decreases to negligible values. Moreover, such influence is weakened under the invariance of the volume fraction of the hard layer and with the thinning of the soft layer because of the multilayer structure. Therefore, for the biocomposite, an ideal stiffening design is an arch-shaped structure with high content, aspect ratio, and overlap ratio of the hard layers.

The rupture model of the MSCB with a strongly bonded interface is similar to that of the PASB, and it does not match other strengthening disciplines. The failure of the MSCB is determined by the relation between the interfacial shear strength and compressive strength of the hard layer. The yield 


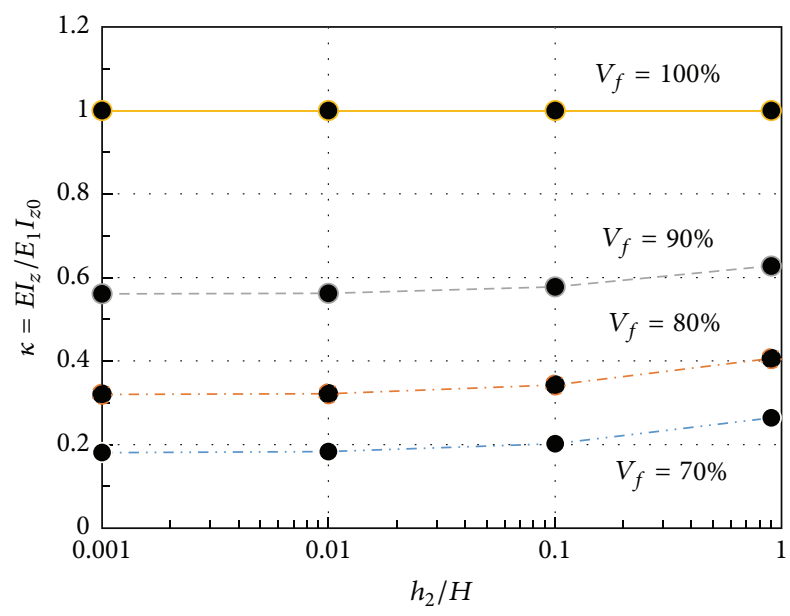

FIGURE 5: Relationship among $\kappa, h_{2} / H$, and $V_{f}$.

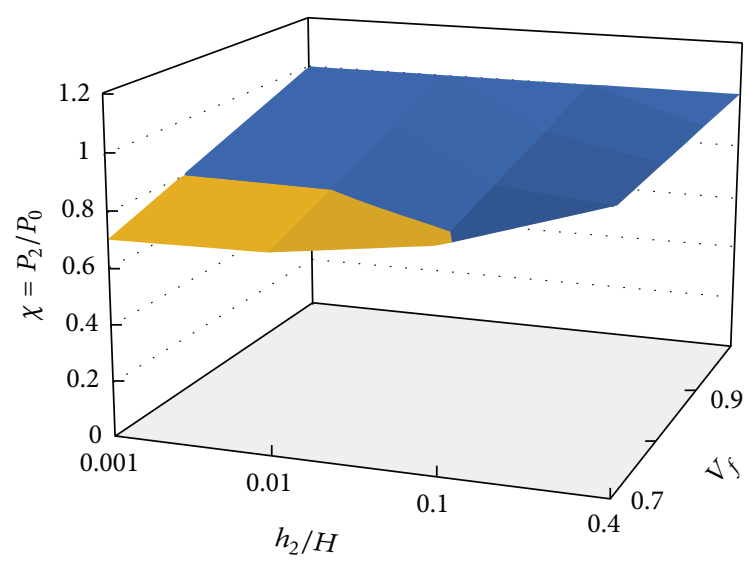

FIgURE 6: Relationship among $\chi, h_{2} / H$, and $V_{f}$.

and failure of the interlayer are the main failure mode of the MSCB with a weak interface; this mode can induce a ductilebrittle transition that is comparable to that of a brittle PASB. The bending strength of the MSCB with a weakly bonded interface decreases with a decrease in the volume fraction of the hard layers and the thickness of the soft layers, as shown in Figure 6. If $V_{f} \geq 70 \%$, the critical load carried by the MSCB is $60 \%$ greater than the critical load carried by the PASB, implying that the bending strength of the MSCB is not markedly influenced by the thickness of the soft layer. The cross section can withstand the bending moment because of the adequate inertia moment when the hard phase content is adequate, thus conforming to the stiffening mechanism. For the MSCB with a weak interface, the bending strength is not markedly reduced by the stress transfer of the soft layers, which carry only shear stress. The nanoscaled soft layers and the representing interfaces play important roles in the strengthening mechanisms of the arch-shaped multilayer structure. The MSCB produces interfacial failure, and plastic deformations proceed along the interlayers when the soft layer begins to yield. The bending strength of the MSCB also decreases with an increase in the interfacial shear strength.
However, the length of the plastic region is amplified by the weaker interface. These findings can serve as basis for creating good strengthening design and for toughening the MSCB.

In consideration of the shearing soft layer, the compressed hard layer, and the arch shape, the unique mechanical properties of the component phases are completely utilized in the MSCB. The MSCB structure with a weak interface starts to deform plastically once the interfacial shear strength is achieved. Thus, the length of the plastic region is closely related to the absorbed energy of the MSCB. The length of the plastic region increases with a decrease in arch height and interfacial shear strength (19). A weaker interface corresponds to a greater plastic deformation yielded by the soft layers. The interfacial cracks advance in a zigzag manner in the plastic region and are arrested by the soft layers. Strengthening and toughening mechanisms, such as crack branch and deflection, can be observed in the MSCB, implying that a greater strain energy is absorbed before the rupture. The fracture energy of the MSCB released by cracks in the plastic region is obtained by using the present study's model (23). Adopting the geometries shown in Figure 1(a) and letting $\rho=12, \Phi=50 \%, V_{f}=95 \%$, and $m=500$ [15], the fracture energy is obtained to be almost $500 \gamma \mathrm{HB}$, which is 500 times larger than that of the PASB. The drawback induced by the weak soft layer is completely compensated by the peculiar arch-shaped multilayer structure that has a larger volume fraction of the hard layers and the nanoscaled soft layers. The bending stiffness, strength, and toughness can be synthetically compared in terms of the geometry and property of the component phases. The fracture energy varies with the effective modulus and the bending strength, as shown in Figure 7. The black dot is located at the upper right region, implying that nacre achieves an ideal combination of bending stiffness, strength (100 MPa [1]), and toughness (1000 J [11]). The fracture energy increases with an increase in the volume fraction and aspect ratio of the hard layer, the effective modulus, and the bending strength. A larger volume fraction of the hard layers corresponds to a greater number of thin soft layers and crack deflections. Reduplicative crack deflections increase the path length, thereby toughening the MSCB. The ductile-brittle transition in the MSCB can be achieved by these strengthening and toughening mechanisms. When the soft phase content is adequate and constant, the nanoscaled thickness and additional interlayers improve the toughness of the MSCB structure with a weak interface when the bending strength is reduced slightly. Therefore, the new design disciplines drawn from the MSCB are peculiar arch shape, internal multilayer structure with a large volume fraction, and aspect ratio of hard layers and weak interface.

\section{Conclusion}

The relationship between the multilayer structure and mechanical properties of arch-shaped seashell has been elucidated by the comparison of two three-point bending beams. The bending stiffness and bending strength of MSCB are not markedly reduced by the introduction of soft layers in spite of the high content and medium overlap ratio of hard layers. The influence of thickness and shear modulus of soft 


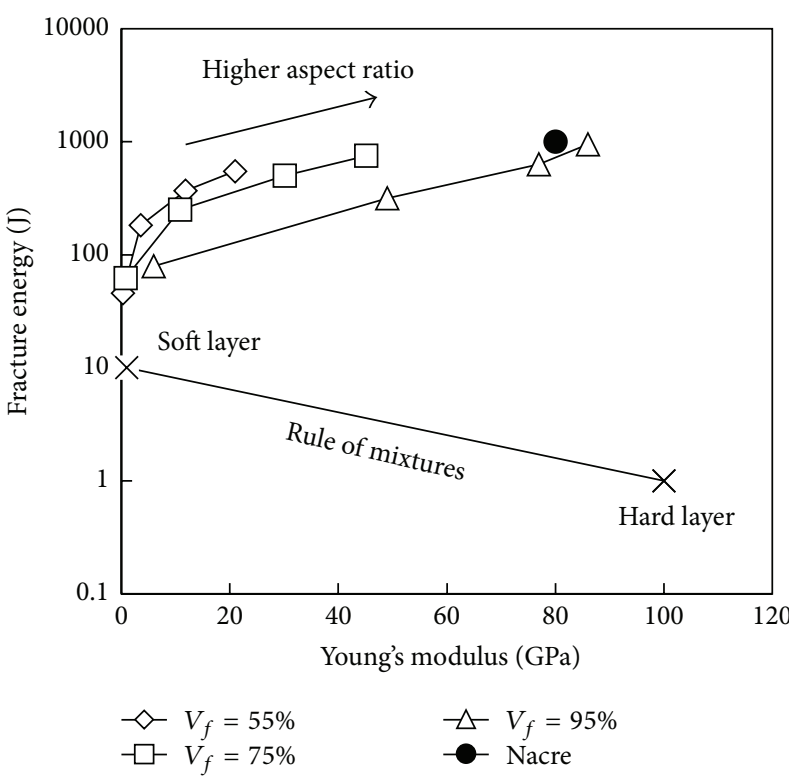

(a) Work of fracture versus effective modulus

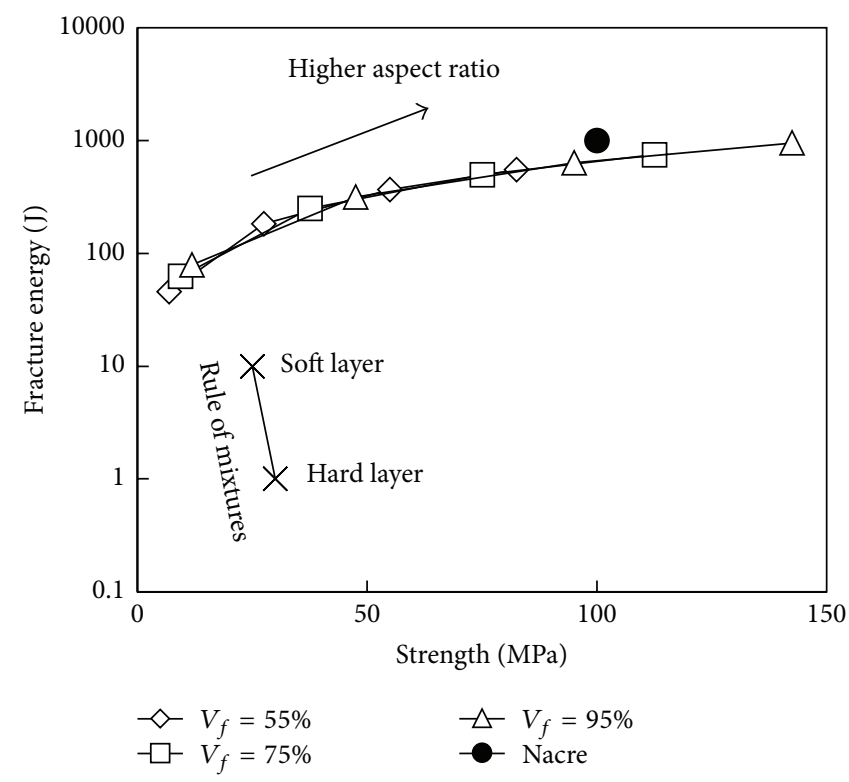

(b) Work of fracture versus bending strength

FIGURE 7: Work of fracture of MSCB $(\Phi=50 \%)$.

layers is gradually decreased to negligible values which lay the foundation for toughening MSCB. The MSCB structure with weak interface starts to deform plastically once the shear strength of the soft layer is achieved. The cracks advance in a zigzag manner in the plastic region of MSCB where more strain energy is absorbed before the rupture. The most important design discipline drawn from the MSCB model is taking advantage of the unique mechanical properties of component phases through the arch shape and multilayer structure. The MSCB structure can be synthetically stiffened, strengthened, and toughened by the peculiar arch shape, internal multilayer structure with larger volume fraction, and aspect ratio of hard layers and nanoscaled soft layers.

\section{Competing Interests}

The authors declare that they have no competing interests.

\section{Acknowledgments}

The authors gratefully acknowledge the financial supports to this work from the National Natural Science Foundations of China (Grant no. 11272367) and Research Project of Sichuan Provincial Department of Education (Grant no. 15ZA0138).

\section{References}

[1] F. Barthelat, "Biomimetics for next generation materials," Philosophical Transactions of the Royal Society A: Mathematical, Physical and Engineering Sciences, vol. 365, no. 1861, pp. 29072919, 2007.

[2] F. Barthelat and R. Rabiei, "Toughness amplification in natural composites," Journal of the Mechanics and Physics of Solids, vol. 59, no. 4, pp. 829-840, 2011.
[3] G. Mayer, "Rigid biological systems as models for synthetic composites," Science, vol. 310, no. 5751, pp. 1144-1147, 2005.

[4] M. Sarikaya, K. E. Gunnison, and M. Yasrebi, "Mechanical property-microstructural relationships in abalone shell," Materials Research Society Symposium Proceedings, vol. 174, pp. 109116,1990

[5] K. Chen, B. Shi, Y. Yue, J. Qi, and L. Guo, "Binary synergy strengthening and toughening of bio-inspired nacre-like graphene oxide/sodium alginate composite paper," ACS Nano, vol. 9, no. 8, pp. 8165-8175, 2015.

[6] S. J. Wan, J. S. Peng, Y. C. Li, H. Hu, L. Jiang, and Q. F. Cheng, "Synergistic toughening of graphene oxidemolybdenum disulfide-thermoplastic polyurethane ternary artificial nacre," ACS Nano, vol. 9, no. 1, pp. 708-714, 2015.

[7] Y. Shao, H.-P. Zhao, X.-Q. Feng, and H. Gao, "Discontinuous crack-bridging model for fracture toughness analysis of nacre," Journal of the Mechanics and Physics of Solids, vol. 60, no. 8, pp. 1400-1419, 2012.

[8] M. R. Begley, N. R. Philips, B. G. Compton, D. V. Wilbrink, R. O. Ritchie, and M. Utz, "Micromechanical models to guide the development of synthetic 'brick and mortar' composites," Journal of the Mechanics and Physics of Solids, vol. 60, no. 8, pp. 1545-1560, 2012.

[9] Y. Ni, Z. Q. Song, H. Y. Jiang, S.-H. Yu, and L. He, “Optimization design of strong and tough nacreous nanocomposites through tuning characteristic lengths," Journal of the Mechanics and Physics of Solids, vol. 81, pp. 41-57, 2015.

[10] C. E. Greene and A. E. Greene, Structural Mechanics, BiblioLife, Charleston, SC, USA, 2008.

[11] H. Gao, "Application of fracture mechanics concepts to hierarchical biomechanics of bone and bone-like materials," International Journal of Fracture, vol. 138, no. 1-4, pp. 101-137, 2006.

[12] C. Jenkins and S. K. Khanna, Mechanics of Materials: A Modern Integration of Mechanics and Materials in Structural Design, Academic Press, Cambridge, UK, 2005. 
[13] A. J. Phillipps, W. J. Clegg, and T. W. Clyne, "Fracture behaviour of ceramic laminates in bending-I. Modelling of crack propagation," Acta Metallurgica et Materialia, vol. 41, no. 3, pp. 805817, 1993.

[14] F. Song, A. K. Soh, and Y. L. Bai, "Structural and mechanical properties of the organic matrix layers of nacre," Biomaterials, vol. 24, no. 20, pp. 3623-3631, 2003.

[15] A. G. Evans, Z. Suo, R. Z. Wang, I. A. Aksay, M. Y. He, and J. W. Hutchinson, "Model for the robust mechanical behavior of nacre," Journal of Materials Research, vol. 16, no. 9, pp. 24752482, 2001. 

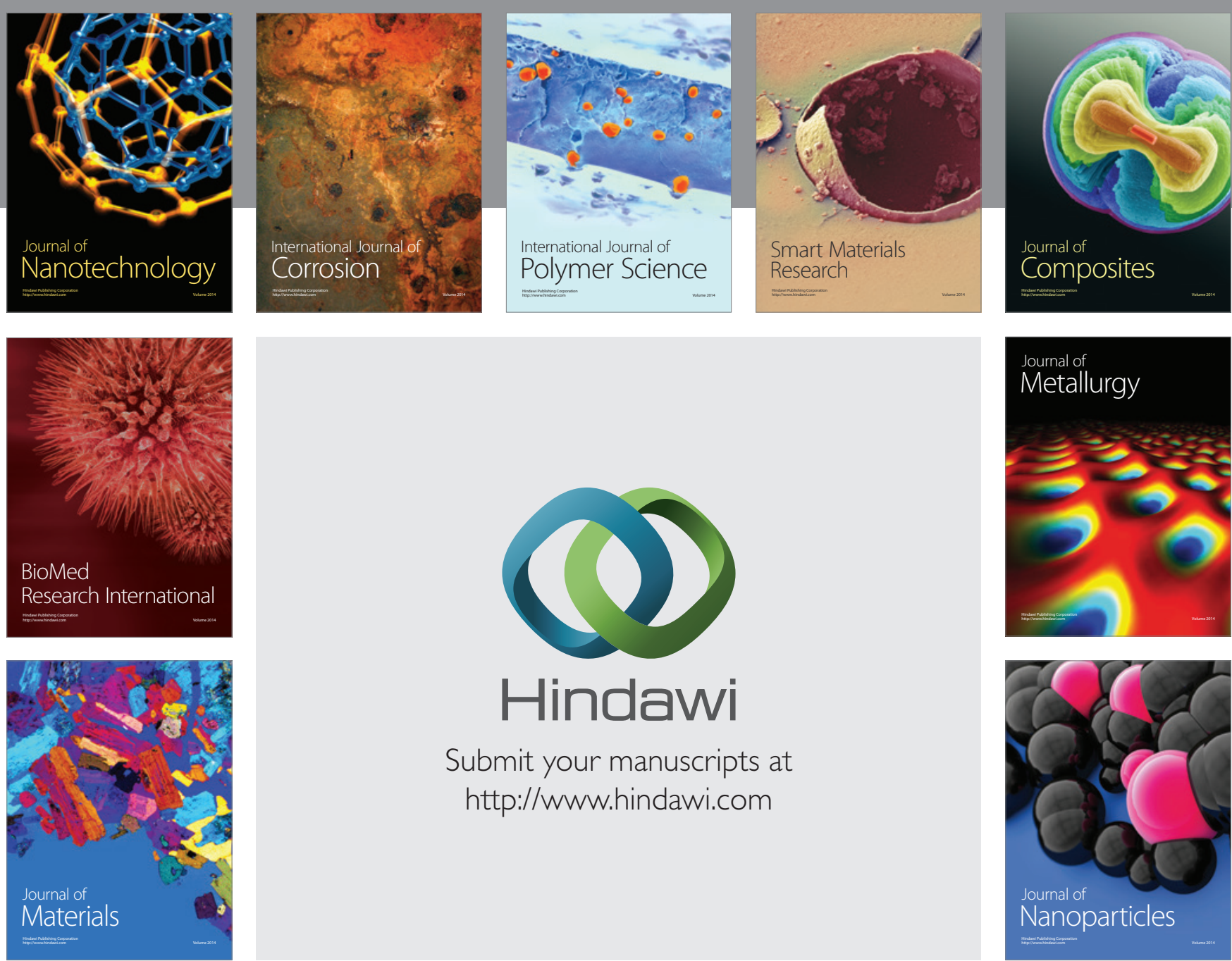

\section{Hindawi}

Submit your manuscripts at

http://www.hindawi.com

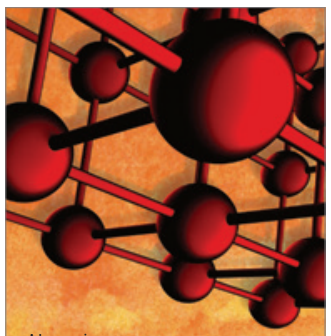

Materials Science and Engineering
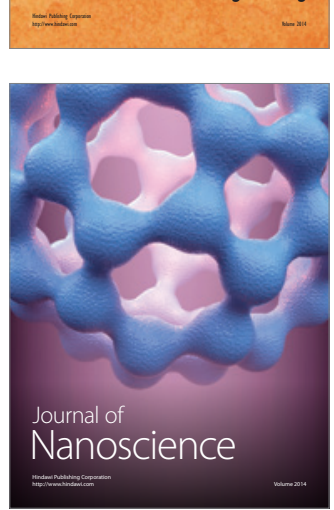
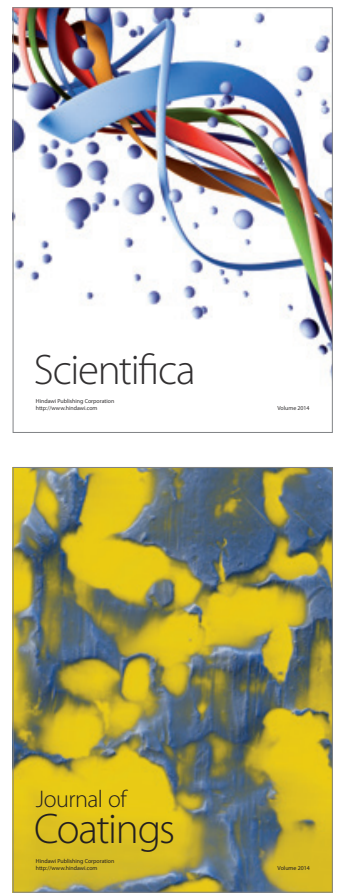
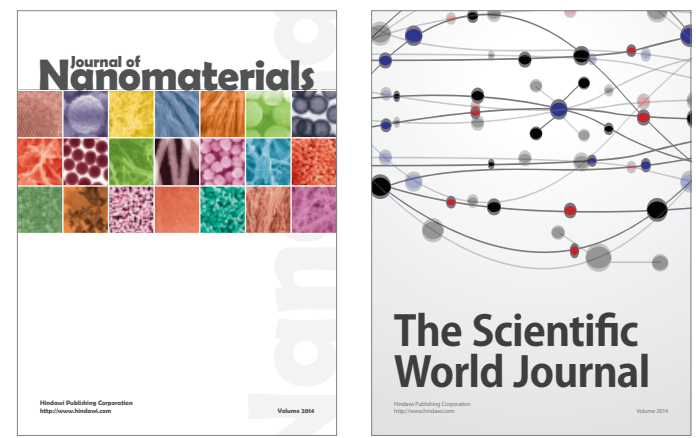

The Scientific World Journal
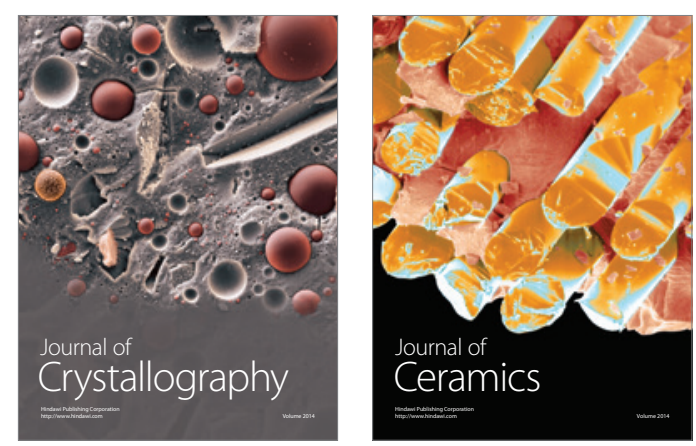
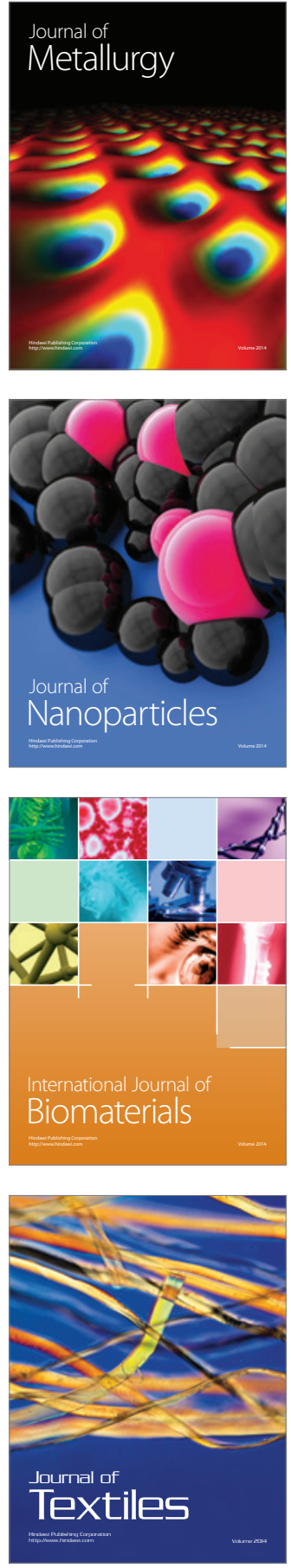